\title{
Comparing orthogonal force and unidirectional strain component processing for tool condition monitoring
}

\author{
Burkhard H. Freyer • P. Stephan Heyns • \\ Nico J. Theron
}

\begin{abstract}
Signal processing using orthogonal cutting force components for tool condition monitoring has established itself in literature. In the application of single axis strain sensors however a linear combination of cutting force components has to be processed in order to monitor tool wear. This situation may arise when a single axis piezoelectric actuator is simultaneously used as an actuator and a sensor, e.g. its vibration control feedback signal exploited for monitoring purposes. The current paper therefore compares processing of a linear combination of cutting force components to the reference case of processing orthogonal components. Reconstruction of the dynamic force acting at the tool tip from signals obtained during measurements using a strain gauge instrumented tool holder in a turning process is described. An application of this dynamic force signal was simulated on a lter-model of that tool holder that would carry a self-sensing actuator. For comparison of the orthogonal and unidirectional force component tool wear monitoring strategies the same time-delay neural network structure has been applied. Wearsensitive features are determined by wavelet packet analysis to provide information for tool wear estimation. The probability of a difference less than 5 percentage points between the ank wear estimation errors of above mentioned two processing strategies is at least $95 \%$. This suggests the viability of simultaneous monitoring and control by using a self-sensing actuator.
\end{abstract}

Keywords Tool wear-monitoring - Self-sensing actuator . Structure dynamic modelling · Neural network .

Wavelet packet analysis

B. H. Freyer $(\bowtie) \cdot$ P. S. Heyns · N. J. Theron

Dynamic Systems Group, Department of Mechanical and Aeronautical Engineering, University of Pretoria, 0002 Pretoria, South Africa e-mail: u04276655@tuks.co.za

\section{Introduction}

In harsh machining circumstances tool condition monitoring (TCM) sensors may have to be embedded or semi-embedded in a tool holder in such a way that they do not interfere with the cutting process. A single axis sensor might then only be able to provide a signal, which is a linear combination of the orthogonal cutting force components. An example of such an application would be a piezoelectric self-sensing actuator (SSA) used as a sensor and an actuator simultaneously in the active control of vibrations. Anderson and Hagood (1994) investigated the principle of the SSA. The signal from the SSA, which is the feedback signal for the vibration control system, could be made available for TCM while simultaneously playing a role in tool vibration control.

In TCM the measurement of its orthogonal components often provides the necessary cutting force information as can be found in the work of Jemielniak et al. (1998), Purushothaman (2010) and Sharma et al. (2008). This work however compares TCM based on orthogonal forces to the one based on measurement of only the unidirectional strain signal. An opportunity for the application of the latter comes with the implementation of the SSA concept in turning processes. The SSA senses the strain caused by a linear combination of the orthogonal cutting force components. With a single-stack SSA it is possible to sense only the unidirectional strain it experiences, so that only the joint effect of the tangential, feed and thrust components of the cutting force can be exploited in TCM and not the effect of the individual components.

The main topic of this work is to explore the feasibility of a monitoring system. This system makes use of a signal, which is a linear combination of the cutting force components. It is compared to a reference case, which is a system using signals of the individual orthogonal force components 
themselves. The focus is therefore on the comparison of two different cases of input signals to the monitoring systems used for the same TCM algorithm in each case. The focus is therefore not on the TCM algorithm itself, since the latter is already known in literature (Scheffer et al. 2003; Sick 2002). This study is also a step towards the implementation of an SSA-based TCM system. The main question pertaining to this monitoring problem is whether the difference in performance between the proposed way of monitoring and the reference case is sufciently small to justify implementation of such a monitoring system.

Neural networks (NN) are commonly used for TCM (Scheffer et al. 2003; Liu and Altintas 1998). In the case of Liu and Altintas (1998) it is argued that their particular $\mathrm{NN}$ algorithm is depended on the robustness and practicality of cutting-force sensors. This is an argument assumed here to be applicable to all NN-type TCM algorithms. This argument favours an investigation into monitoring done with a piezoelectric transducer, semi-embedded underneath the tool holder. The research on the simultaneous monitoring of tool condition and vibration control capabilities may improve the versatility of the tool holder and may also pave the way for a fully embedded transducer. This could mean almost eliminating its interference with the performance of the tool, which is important in this context as Lundholm et al. (1988) have put it.

Literature reports on various measurement systems, depending on the type of measured variable for TCM. When cutting force is the measured variable for TCM, it is often acquired by means of dynamometers (Bahre et al. 1992; Seah et al. 1995; Audy 2006). Others have used accelerometers to measure tool vibration (Bonifacio and Diniz 1994; Abouletta and $\mathrm{M} \square \mathrm{dl} 200$ ) whereas methods based on strain gauges are used for measuring either cutting forces as in Scheffer et al. (2003) or vibrations as in Li and Ulsoy (1999). Papers by Cho et al. (1999) as well as Sick (2002) show that cutting force is the process variable that is most commonly used for tool wear monitoring. It was therefore decided to measure cutting forces using a strain gauge instrumented tool holder.

The signals sensed with the strain gauge instrumented tool holder were converted into the dynamic force acting at the tool tip. In aid of this conversion was a modal state space model of this tool holder. A Laplace domain model of the tool holder for use with the SSA supplied the parameters when the dynamic force signal was ltered to simulate the correct strain signal that would be measured by the SSA.

Work by Sick (2002), Scheffer et al. (2003), Choudhury and Bartarya (2003) as well as Liu and Altintas (1998) was used as the basis for the tool wear-monitoring aspects pertaining to the use of NNs. The use of wavelet packet analysis for the computation of tool wear sensitive features was adopted from Wu and Du (1996) and Pal et al. (2011). Freyer et al. (2008) have already suggested vibration control in a turning process using an SSA. This paper discusses how a linear combination of the force components is associated with their use in TCM.

The following section shows how the force component, to be used for the SSA involved TCM, can be reconstructed from signals of a strain gauge instrumented tool holder. The content of the section on data acquisition, describes an experiment conducted to obtain data on cutting forces and ank wear. The sections on feature selection and data-processing methods then follow with the latter containing the articial intelligence basis of the TCM. A statistical presentation of the results as well as accuracy of the TCM systems are then given in the next section and the nal section concludes this work. This means that the actual signal processing for TCM purposes is only contained in the section on data-processing, while the preceding sections describe measurements and modelling performed to provide the data for this investigation, however as mentioned above the objective of this investigation is a comparison of TCM based on orthogonal versus unidirectional sensed signals.

\section{Reconstruction of unidirectional sensed strain signal}

\section{Modelling problem definition}

The SSA tool holder conguration shown in Fig. 1 represents the conguration considered here for investigating the concept of simultaneous tool vibration control and TCM. The signals that are normally desired for TCM purposes are the components $f_{1 x}, f_{1 y}$ and $f_{1 z}$ of the cutting force $f_{1}$, acting at node 1, as shown in Fig. 1. However, the SSA can only sense its own axial strain, which results from the external forces and also the voltage applied to it as elaborated in Anderson and Hagood (1994). The displacements $u_{1 x}(t)$ and $u_{2 z}(t)$ of the tool holder in Fig. 1 are a result of all the forces acting on this structure, namely $f_{1}$ and $f_{2}=f_{2 z}$. Due to the voltage that would normally be applied to the SSA it exerts the actuation force $f_{2 z}$ on the tool holder at node 2 , as indicated in Fig. 1. It is assumed that there is no displacement at node 3 and that the SSA can therefore be assumed being grounded at this node. In the Laplace domain, $u_{1 x}(t)$ and $u_{2 z}(t)$ can be represented by the following relationships:

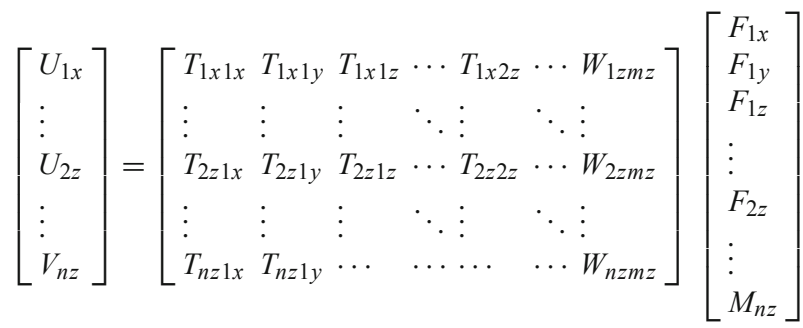


Fig. 1 Tool holder and SSA with degrees of freedom indicated

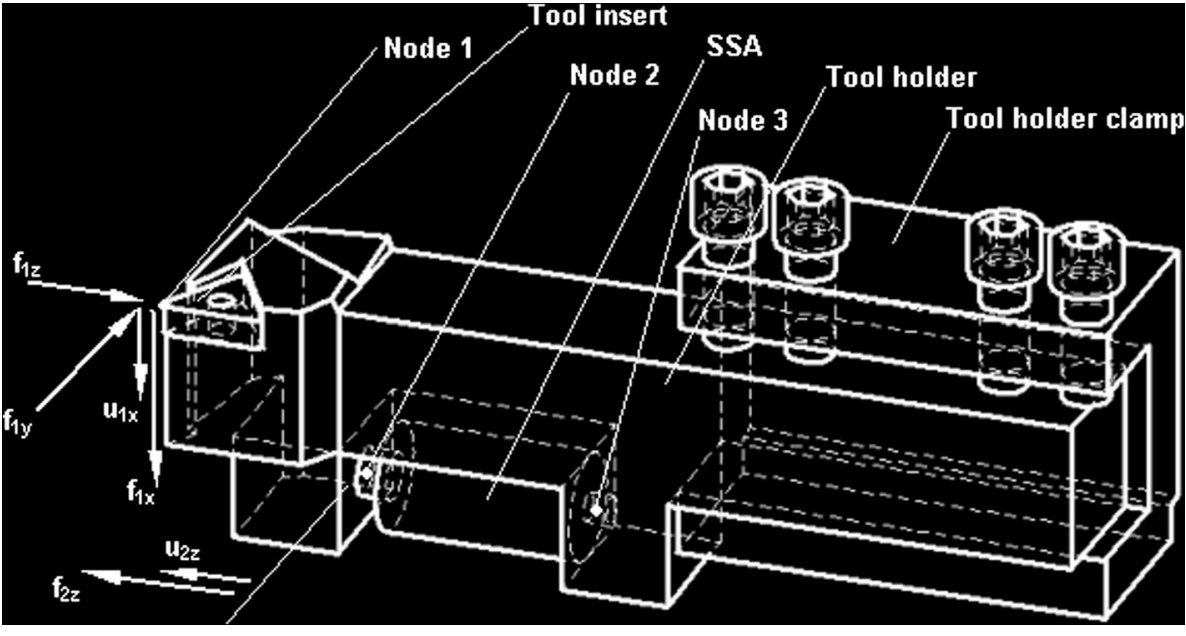

where models $T_{i v j w}$ represent force $F_{j w}$ to translation $U_{i v}$ or rotation $V_{i v}$ transfer functions and $W_{i v j w}$ represent moment $M_{j w}$ to translation $U_{i v}$ or torsion $V_{i v}$ transfer functions. Their subscripts $i$ and $j$ refer to the nodes of output and input respectively. Subscripts $v$ and $w$ refer to the $\mathrm{x}-, \mathrm{y}$ - or $\mathrm{z}$-axis of the corresponding output displacement and input force respectively. It is here dealt with the inuence of $f_{1}(t)$ on $u_{2 z}(t)$ only i.e. with the SSA as a passive element of the tool holder structure. With $f_{2 z} \neq 0$, the SSA would be active. From Eq. (1) only displacements $U_{1 x}$ and $U_{2 z}$ will furthermore be considered and the displacements and rotations at the remaining nodes be discarded.

A model of the Mitsubishi DTGNR 2525 M16-type tool holder was created in MD Patran. This tool holder and the brackets, between which the SSA is mounted (Fig. 1), are modelled as one solid part. It was meshed with 91361 tet10 elements, which had edge lengths of typically $2 \mathrm{~mm}$. The SSA was modelled as a 1 -D bar with a mechanical stiffness $K_{a}$ equal to that of the Piezomechanik GmbH-type PSt 500/10/15 VS 18 piezoelectric stack actuator.

In this work $f_{1}(t)$ has been determined from signals sensed by tool holder equipped with strain gauges as shown in Fig. 2. The strain gauges of the half bridges had a length of $3 \mathrm{~mm}$ and of $6 \mathrm{~mm}$ in the full bridge, which make measurement at least up to frequencies such as $20 \mathrm{kHz}$ possible according to a relationship between peak strain and frequency for a certain gauge length. The paper of Swantek et al. (2001) contains such a relationship.

The tool holder in Fig. 2, a Seco PTJNR-2020-16A, was modelled as a cantilever clamped as shown in Fig. 3. It was modeled with 6312 hex- 8 elements in a nite element method (FEM), such that the cross section of $20 \times 20 \mathrm{~mm}$ consisted of $10 \times 10$ elements. The tool holder had an overhang of $69.7 \mathrm{~mm}$ and those nodes corresponding to the points of contact when clamped in a tool post of a Colchester Student lathe were constrained in directions of translation and rotation. The strain gauge protection was modelled as a point mass

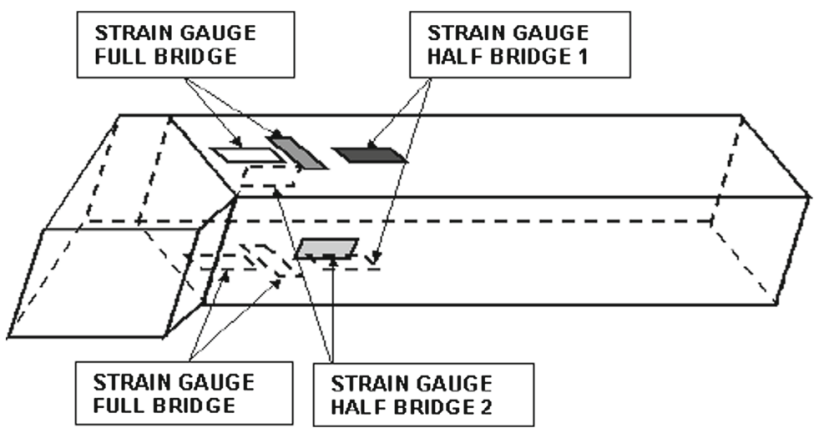

Fig. 2 Strain gauge (SG) instrumented tool holder with SG1 darkgray, SG2 light-gray and SG3 gray

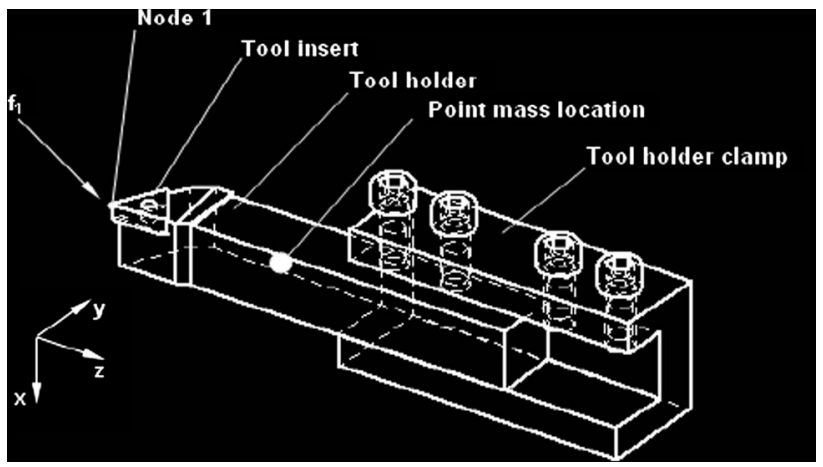

Fig. 3 Strain gauge instrumented tool holder as clamped

$m_{p m}=0.58196 \mathrm{~kg}$ located at the centre of this protection. The reason for this particular value lies in the requirement to set the frequency of the rst bending mode of the model equal to the equivalent frequency observed on the real strain gauge instrumented tool holder. This setting was done by adjustment of $m_{\mathrm{pm}}$.

The strain gauge half bridges were placed at a position where sufcient strain due to bending could be sensed. In order to let this bending happen an abnormally long overhang of the tool holder was chosen. 
To be able to reconstruct the dynamic force at the tool tip one only has the modal properties of the tool holder and measurements of three strain gauge bridges. In order to have a model linking displacement to input force and with the absence of mass stiffness and damping matrices, it is convenient to work in the modal domain and then also with some input-output $(\mathrm{I} / \mathrm{O})$ model of which one can transform the output to a variable with physical meaning.

Without two operations, one being an I/O model having forces as input and modal coordinates, as output and successive transformation from modal to physical coordinates the modelling problem would be more complicated. An I/O model however can be formulated to make the required variable accessible at the output, e.g. in state space formulation, as given in Balm $\square \mathrm{s}$ and Lecl $\square \mathrm{re} 2003$ ). Even though only 3 inputs and outputs are required in this work the latter is a manageable type of a multivariable model and has advantages over e.g. Laplace domain models especially for higher numbers of $\mathrm{I} / \mathrm{O}$ variables.

A reconstruction of $f_{1}(t)$ from the available strain gauge measurement was therefore done by means of a multi-inputmulti-output (MIMO) state space formulation of the structural dynamics in the modal domain. Even though a model in the modal domain, physical displacements become accessible at the output by means of a transformation. Likewise strains can be made accessible by means of transformation with a strain eigenvector. Its relationship with displacement modes is shown in the following subsection.

\section{Modeling problem description}

The following symbols are among those used for the formulation of a model, which has strain measurements as inputs and dynamic force components at the tool tip as outputs.

$p_{j}$ : modal coordinate corresponding to mode $j$

$p_{j}^{\varepsilon}$ : $\quad$ strain modal coordinate corresponding to mode $j$

$\boldsymbol{x}: \quad n \times 1$ physical coordinate, such that $\boldsymbol{x}=\boldsymbol{\Phi} \boldsymbol{p}$

$\boldsymbol{u}: \quad n \times 1$ displacement vector

$r: \quad n \times 1$ position vector

$\boldsymbol{\varepsilon}$ : strain tensor

$\boldsymbol{\Phi}: \quad n \times m$ modal matrix with displacement eigenvectors as columns, $n$ DOFs and $m$ modes

$\phi_{j}: \quad j$ th normal mode of the system

$\phi_{j i}$ : $\quad i$ th entry of $j$ th normal mode of the system

$\psi_{j}$ : $\quad$ strain eigenvector tensor for mode $j$

$\psi_{j k l}$ : component $(k, l)$ of strain eigenvector tensor for mode $j$

$\boldsymbol{\Omega}^{2}: \quad \boldsymbol{\Omega}^{2}=\operatorname{diag}\left(\boldsymbol{\omega}_{i}^{2}\right), \quad i=1,2, \ldots m$, where $m$ is the number of modes

$\boldsymbol{\Gamma}: \quad \boldsymbol{\Gamma}=2 \boldsymbol{\xi} \boldsymbol{\Omega}$, where $\boldsymbol{\xi}=\operatorname{diag}\left(\boldsymbol{\xi}_{i}\right), i=1,2, \ldots m$

$\boldsymbol{E}_{S 2}$ : vector of three Laplace domain strain outputs at selected DOFs, set $S 2$ of DOFs

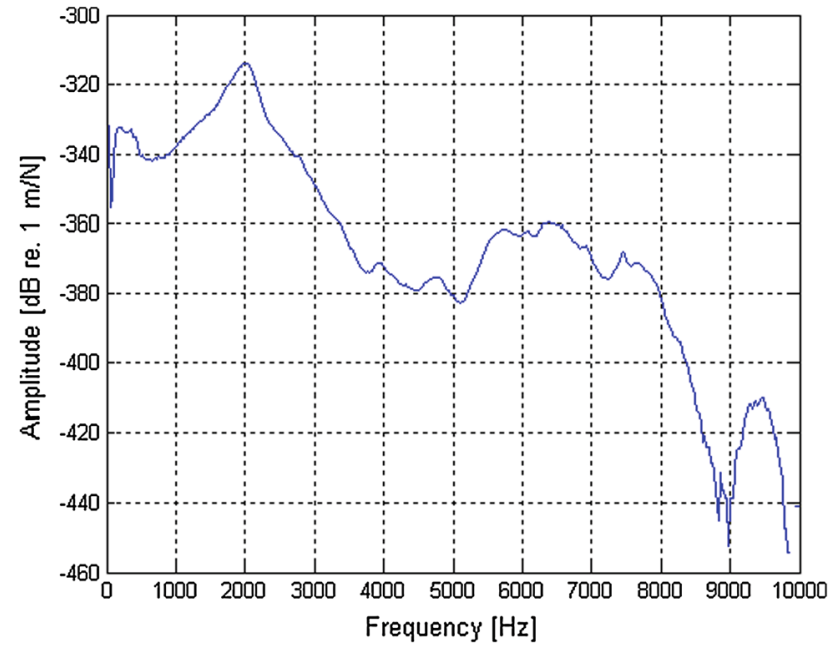

Fig. 4 Frequency response from strain gauge instrumented tool holder when clamped with force and displacement at tool tip as input and output respectively, both in $x$-direction

$\boldsymbol{F}_{S 1}$ : vector of three Laplace domain input force components for translation DOFs at tool tip, set $S 1$ of DOFs

b: $\quad n \times 3$ locating matrix with $b_{i j}=1$, for translation DOFs at tool tip, set $S 1$ of DOFs

c: $\quad 3 \times n$ locating matrix with $c_{i j}=1$, at DOFs for selected outputs, set $S 2$ of DOFs

$\xi_{i}$ is the viscous modal damping and $\omega_{i}$ the natural frequency at mode $i$. For the rst ve modes the damping has been determined from the percentage overshoot (Dorf and Bishop 2005) of the tool tip at each mode during a sudden release test of the tool holder in Fig. 3. $\xi_{1}$ to $\xi_{3}$ have been determined as $0.4468,0.4605$, and 0.4076 while $\omega_{1}$ to $\omega_{3}$ were found at 1.978, 2.1 and $4.169 \mathrm{kHz}$ from FEM software MD Nastran, where $\omega_{2}$ corresponds to the rst bending mode of this tool holder around the y-axis. The bending mode is also shown in the frequency response function of Fig. 4 . Since the eigenvectors are produced in a normal mode solution type in Nastran we can write the following state-space representation of our normal mode model:

$$
\begin{aligned}
& \left\{\begin{array}{l}
\dot{p} \\
\ddot{p}
\end{array}\right\}=\left[\begin{array}{cc}
0 & \boldsymbol{I} \\
-\boldsymbol{\Omega}^{2} & -\Gamma
\end{array}\right]\left\{\begin{array}{l}
\boldsymbol{p} \\
\dot{\boldsymbol{p}}
\end{array}\right\}+\left[\begin{array}{c}
\boldsymbol{0} \\
\boldsymbol{\Phi}^{T} \boldsymbol{b}
\end{array}\right]\{\boldsymbol{f}(t)\} \\
& \{\boldsymbol{u}(t)\}=\left[\begin{array}{ll}
\boldsymbol{c} \boldsymbol{\Phi} & 0
\end{array}\right]\left\{\begin{array}{l}
\boldsymbol{p} \\
\dot{\boldsymbol{p}}
\end{array}\right\}
\end{aligned}
$$

Our problem is as follows. In Eq. $(2 \mathrm{a}, \mathrm{b})$ we have three timevarying input force components at three DOFs of the tool tip and three time varying displacements at the output $\boldsymbol{u}(t)$. Pisoni et al. (1995) show that from the relationship of the strain tensor at each position to the displacements in the structure, 


$$
\begin{aligned}
\boldsymbol{\varepsilon}(\boldsymbol{r}, t) & =\frac{1}{2}\left(\frac{\partial \boldsymbol{u}}{\partial \boldsymbol{x}}+\left[\frac{\partial \boldsymbol{u}}{\partial \boldsymbol{x}}\right]^{T}\right) \\
& =\sum_{j=1}^{\infty} \frac{1}{2}\left(\frac{\partial \boldsymbol{\phi}_{j}(\boldsymbol{r})}{\partial \boldsymbol{x}}+\left[\frac{\partial \boldsymbol{\phi}_{j}(\boldsymbol{r})}{\partial \boldsymbol{x}}\right]^{T}\right) p_{j}(t) .
\end{aligned}
$$

An equivalent relationship of the strain eigenvector tensor $\psi_{j}(\boldsymbol{r})$ for mode $j$ and at each position of the system in terms of the $j^{\prime}$ th normal mode, $\boldsymbol{\phi}_{j}$, of the system as

$\boldsymbol{\psi}_{j}(\boldsymbol{r})=\frac{1}{2}\left(\frac{\partial \boldsymbol{\phi}_{j}(\boldsymbol{r})}{\partial \boldsymbol{x}}+\left[\frac{\partial \boldsymbol{\phi}_{j}(\boldsymbol{r})}{\partial \boldsymbol{x}}\right]^{T}\right), \quad j=1, \ldots \infty$

The strain eld in the structure is given in terms of the strain eigenvector tensors as

$\boldsymbol{\varepsilon}(\boldsymbol{r}, t)=\sum_{j=1}^{\infty} \boldsymbol{\psi}_{j}(\boldsymbol{r}) p_{j}^{\varepsilon}(t)$

Pisoni et al. (1995) show that modal coordinates corresponding respectively to strain and displacement can be set equal. Then Eq. (5) becomes

$\boldsymbol{\varepsilon}(\boldsymbol{r}, t)=\sum_{j=1}^{\infty} \boldsymbol{\psi}_{j}(\boldsymbol{r}) p_{j}(t)$

If the position vector $\boldsymbol{r}_{m}$ indicates the strain tensors at positions of the strain gauges, Eq. (6) becomes

$\boldsymbol{\varepsilon}\left(\boldsymbol{r}_{m}, t\right)=\sum_{j=1}^{\infty} \boldsymbol{\psi}_{j}\left(\boldsymbol{r}_{m}\right) p_{j}(t)$

The components $(k, l)$ of the strain eigenvector tensor, $\boldsymbol{\psi}_{j}\left(\boldsymbol{r}_{m}\right)$, can be used to determine the strain tensor, $\varepsilon_{k l}\left(\boldsymbol{r}_{m}, t\right)$, at the position indicated by $\boldsymbol{r}_{m}$ with the relationship

$\varepsilon_{k l}\left(\boldsymbol{r}_{m}, t\right)=\sum_{j=1}^{\infty} \boldsymbol{\psi}_{j k l}\left(\boldsymbol{r}_{m}\right) p_{j}(t)$,

where $\psi_{j k l}\left(\boldsymbol{r}_{m}\right)$ is component $(k, l)$ of the strain eigenvector tensor $\boldsymbol{\psi}_{j}\left(\boldsymbol{r}_{m}\right)$.

In this study however only the normal strains are considered and therefore only those components for which $k=l$, i.e. those on the diagonal of the strain tensor $\varepsilon\left(\boldsymbol{r}_{m}, t\right)$. For a specic strain gauge with orientation along DOF $d$, the strain measurement output would be

$\varepsilon_{d}=\sum_{j=1}^{3}\left(\frac{\partial \phi_{j d}}{\partial x_{d}}\right) p_{j}(t)=\varepsilon_{k l}=\sum_{j=1}^{3} \psi_{j k l} p_{j}(t)$,

with $(d, k, l) \in\{(x, 1,1),(y, 2,2),(z, 3,3)\}$

where $x, y$ and $z$ represent the three translation DOFs at that point. Therefore $\boldsymbol{\psi}_{j d}(\boldsymbol{r})$ is denoted as a vector consisting of the diagonal entries of the strain eigenvector tensor $\boldsymbol{\psi}_{j}(\boldsymbol{r})$ for mode $j$ with $j$ running to 3 only, i.e. for the rst three modes considered. Each entry of $\boldsymbol{\psi}_{j d}(\boldsymbol{r})$ is associated with a translation DOF. Here $\boldsymbol{\Psi}_{D}$ is then denoted as a matrix with the vectors $\psi_{j d}$ as columns, where each element of $\psi_{j d}$ is associated with one of the DOFs of the structure.

State space model Eq. $(2 \mathrm{a}, \mathrm{b})$ then becomes

$$
\begin{aligned}
& \left\{\begin{array}{l}
\dot{\boldsymbol{p}} \\
\ddot{\boldsymbol{p}}
\end{array}\right\}=\left[\begin{array}{cc}
\boldsymbol{0} & \boldsymbol{I} \\
-\boldsymbol{\Omega}^{2} & -\Gamma
\end{array}\right]\left\{\begin{array}{l}
\boldsymbol{p} \\
\dot{\boldsymbol{p}}
\end{array}\right\}+\left[\begin{array}{c}
\boldsymbol{0} \\
\boldsymbol{\Phi}^{T} \boldsymbol{b}
\end{array}\right]\left\{\boldsymbol{f}_{S 1}(t)\right\} \\
& \left\{\boldsymbol{\varepsilon}_{S 2}(t)\right\}=\left[\begin{array}{ll}
c \boldsymbol{\Psi}_{D} & 0
\end{array}\right]\left\{\begin{array}{l}
\boldsymbol{p} \\
\dot{\boldsymbol{p}}
\end{array}\right\},
\end{aligned}
$$

which is of the form

$$
\begin{aligned}
\{\dot{\boldsymbol{z}}(t)\} & =\boldsymbol{A}\{\boldsymbol{z}(t)\}+\boldsymbol{B}\{\boldsymbol{f}(t)\} \\
\{\boldsymbol{\varepsilon}(t)\} & =\boldsymbol{C}\{\boldsymbol{z}(t)\},
\end{aligned}
$$

where

$$
\{z(t)\}=\left\{\begin{array}{c}
p \\
\dot{p}
\end{array}\right\}
$$

Equation $(10 \mathrm{a}, \mathrm{b})$ can be written in Laplace domain as

$\boldsymbol{E}_{S 2}(s)=\left[\boldsymbol{C}(s \boldsymbol{I}-\boldsymbol{A})^{-1} \quad \boldsymbol{B}\right] \boldsymbol{F}_{S 1}(s)=\boldsymbol{H}(s) \boldsymbol{F}_{S 1}(s)$.

High frequency effects were added to the entries of rational matrix $\boldsymbol{H}(s)$ in terms of zeros at $s=-20,000$ and $s=$ $-25,000$ in order to make them bi-proper. This was necessary to obtain a transmissibility matrix $\boldsymbol{D} \neq 0$ for $s \rightarrow \infty$ (Skogestad and Postlethwaite 2001). Following this modication a state space realization was obtained from the modied rational matrix $\boldsymbol{H}_{n}(s)$ aided by the Control System Toolbox from Mathworks (2002a). From the resulting state space realization an inverse realization was obtained as the quadruple

$$
\left[\begin{array}{ll}
\boldsymbol{A}_{n} & \boldsymbol{B}_{n} \\
\boldsymbol{C}_{n} & \boldsymbol{D}_{n}
\end{array}\right]=\left[\begin{array}{cc}
\boldsymbol{A}-\boldsymbol{B} \boldsymbol{D}^{-1} \boldsymbol{C} & \boldsymbol{B D}^{-1} \\
-\boldsymbol{D}^{-1} \boldsymbol{C} & \boldsymbol{D}^{-1}
\end{array}\right]
$$

Along with

$$
\begin{aligned}
\boldsymbol{F}_{D}(s) & =\boldsymbol{F}_{S 1}(s)=\left[\boldsymbol{C}_{n}\left(s \boldsymbol{I}-\boldsymbol{A}_{n}\right)^{-1} \boldsymbol{B}_{n}+\boldsymbol{D}_{n}\right] \boldsymbol{E}_{S 2}(s) \\
& =\boldsymbol{G}(s) \boldsymbol{E}_{S 2}(s)
\end{aligned}
$$

the dynamic forces at the tool tip can now be determined from the corresponding measured elastic force components.

Equation (3) requires that partial derivatives, specifically directional derivatives in 3 dimensions be computed numerically. For this purpose the software FreeFEM3D from Del Pino and Pironneau (2008) was utilized. The input les for FreeFEM3D had to contain geometry information of the tool holder, which originated from the MD Patran model of the tool holder shown in Fig. 3. These input les were generated with the aid of custom made m-les from Matlab ${ }^{\circledR}$ R13 as well as gmsh software from Geuzaine and Remacle (2009). Visualization of the geometry information contained in the input les was possible through Medit software from Frey (2001). 
Table 1 Experimental equipment

\begin{tabular}{ll}
\hline & Machine, tools and work piece \\
\hline Lathe & Colchester student 1800 (manually operated) \\
Tool holder & SECO PTJNR-2020-16A \\
Insert & Mitsubishi TNGG 160408R $\square$ Carbide UTi20T \\
Work piece material & EN19 oil-quenched, tempered to T-condition, same batch, 269 $\square$ 285 BHN \\
and properties & hardness, 900 $\square 965$ MPa UTS \\
Work piece dimensions & $\square$ 100 $\square 89$ mm $\times$ length 500 mm \\
Work piece surface & Pre-cutting with 0.4 mm nose radius inserts and 0.25 $\square 0.28 \mathrm{~mm} /$ rev feed rate \\
\hline & Instrumentation \\
\hline Feed force sensing & HBM strain gauge, 3/120ALY41, half-bridge \\
Tangential force sensing & HBM strain gauge, 3/120ALY41, half-bridge \\
Thrust force sensing & HBM 90 rosette strain gauge, 1-XY31-6/120, full-bridge \\
Flank wear measurement & Kyowa microscope, magnication: 40, 26 $\mu$ m accuracy \\
Data acquisition system & eDAQ lite by SOMAT \\
Anti-aliasing & eDAQ built-in 6,667 Hz linear phase LP lter \\
Data storage & PC, Pentium 4, with SOMAT test control environment (TCE) \\
& and Ineld software \\
\hline
\end{tabular}

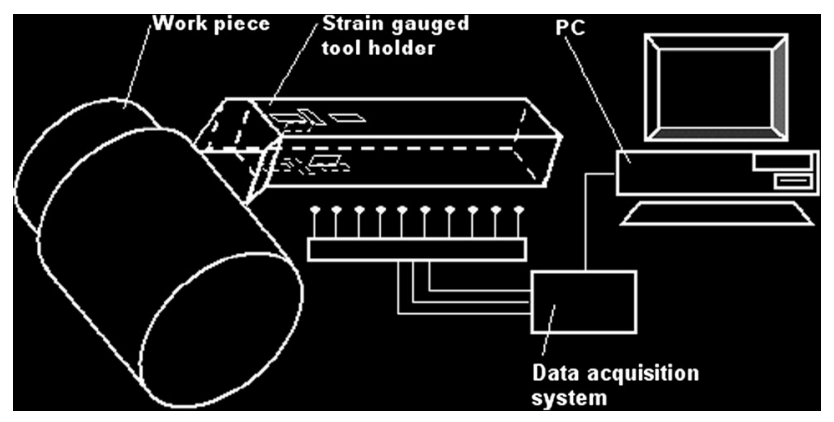

Fig. 5 Experimental set-up

\section{Data acquisition}

\section{Experimental set-up}

A turning experiment was conducted to measure the three components of a cutting force using the type of tool holder shown in Fig. 2 and instrumented with strain gauges as was mentioned in $\square$ Modelling problem definition $\square$ section. These signals were acquired such that they could be used to compare conventional strain gauge based TCM by sensing the cutting force components with TCM based on sensing of a single SSA signal. A schematic diagram of the experimental set-up is shown in Fig. 4. The equipment used for this work is listed in Table 1 (Fig. 5).

Pre-cutting was done with a $0.4 \mathrm{~mm}$ nose radius for the insert, however a larger radius, in this case $0.8 \mathrm{~mm}$, was selected here so that the tool would vibrate with larger amplitudes and consequently better exploit the resolution capabilities of the acquisition system $\square$ s range setting.

\section{Experimental procedure}

The experiment was designed in the nish cutting range based on Mitsubishi (2007) so that measurements could be taken and monitoring done at different machining conditions, consisting of all possible combinations of the following settings for cutting speed (CS) in $\mathrm{m} / \mathrm{min}$, depth of cut (DOC) in $\mathrm{mm}$ and feed rate (FR) $\mathrm{mm} / \mathrm{rev}$ :

$$
\left.\begin{array}{l}
C S_{i} \in\{128.8,119.8,114.6\} \\
D O C_{i} \in\{1.2,1.4,1.6\} \\
F R_{i} \in\{0.1,0.16,0.2\}
\end{array}\right\} \text { for } i=1,2,3
$$

The selection of the specic insert and these particular cutting parameters was inuenced by a number of requirements, which had to be met. They were

i. observable progression of ank wear, hence the choice of a uncoated carbide insert

ii. fast tool wear, in order to speed up experiment and reduce the number of work pieces needed

iii. cutting forces should cause measurable deection of tool holder

iv. the selected values of the cutting parameter should have sufcient spread to give statistical condence for a model derived from quadratic regression mentioned in a section about the accuracy of the TCM systems. 


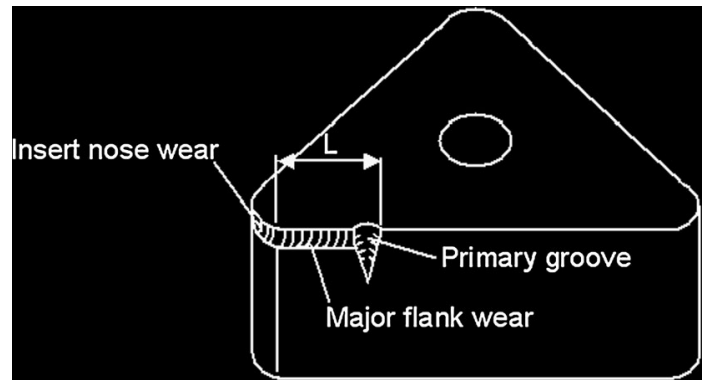

Fig. 6 Measurement of ank wear

The inuences of the insert type on chip formation and also its resulting effect on cutting force is not object of this study. The same insert types were used in the cutting process from which the cutting force signals used for this study originated.

Chen et al. (1993) describe how the state of the work piece surface before cutting, critically inuences the cutting forces. Therefore the work pieces were pre-cut to keep their surface condition approximately constant.

The discrete measurements included the diameter of the work piece before and after a pass, the axial length, the duration of each pass and the photographic capture of insert ank wear. True values for CS and DOC could then be determined for every pass. The true FR of the lathe was determined by means of a dial gauge to $0.01 \mathrm{~mm} / \mathrm{rev}$ accuracy. Flank wear was measured from the photographs as $V B=A / L$, where $V B$ is the ank wear in mm and $A$ is the area of the major ank wear, including the area of the primary groove (see Fig. 6).

The variable error of each ank wear measurement is between -0.0006 and $+0.0006 \mathrm{~mm} \square$ this error can be different for each VB-measurement and is based on accuracy of the ruler used to do the measurements. The constant, i.e. the consistent error is between -0.026 and $+0.026 \mathrm{~mm} \square$ this error is the same for all VB-measurements and is based on the microscope accuracy.

Continuous measurements comprised the acquisition of three signals from a strain-gauged tool holder by a data acquisition system at a sampling frequency of $20 \mathrm{kHz}$. The instrumentation of the tool holder was designed so that it could sense bending by tangential and feed forces and also the longitudinal deformation caused by the thrust force.

A calibration matrix $\boldsymbol{A}$ was determined to uncouple the interaction between the actual forces and the measured signals on each channel by means of transformation of coordinates.

The coefcients $a_{i j}$ of this matrix are the ratios of voltages $v_{i}, i \in[1,2,3]$ measured at each of the three channels of the strain gauge instrumented tool holder to a force $f_{j}$ applied in one of the three directions (tangential, feed and thrust) at the tool tip, while $f_{l}=0$ with $l \neq j$ and $j, l \in\left[\begin{array}{ll}x, y, z & \text {, }\end{array}\right.$ i.e. $a_{i j}=\frac{v_{i}}{f_{j}}$. Each $a_{i j}$ was averaged over three sets of force and voltage values.
Post-processing of the signals consisted of multiplication of the inverse calibration matrix in order to obtain the three orthogonal force components from the three voltage signals, i.e. at time instant $k$,

$\boldsymbol{f}_{k}=\boldsymbol{A}^{-1} \boldsymbol{v}_{k}$

where $\boldsymbol{f}_{k}=\left[f_{x_{k}} f_{y_{k}} f_{z_{k}}\right]^{T}$ and $\boldsymbol{v}_{k}=\left[\begin{array}{lll}v_{1_{k}} & v_{2_{k}} & v_{3_{k}}\end{array}\right]^{T}$.

With Eq. (16) the inputs for the orthogonal TCM system were produced. The following signal conditioning however had to be done to obtain the inputs to the unidirectional TCM system. As inputs to the model in Eq. (15), the true strain values at selected nodes, with the dynamic force components at the tool tip as outputs, had to be obtained. These nodes were selected to coincide with SG1, SG2 and SG3 in Fig. 2.

Two types of causes of combined loads on strain gauges were considered. One of them results in strain gauges measuring combined loads such as SG1 and SG2 in Fig. 2. They sense strain due to bending around the $\mathrm{y}$ - and $\mathrm{x}$-axis respectively and are also affected by the axial load along the z-axis. However the second cause of combined load sensing is due to alignment errors of the individual strain gauges on the tool holder. Such errors occur where a half bridge, which was designed to sense only bending around a certain axis, is to some degree also sensitive to bending around another axis or to axial thrust. The effect of thrust on half bridges, which are by design bending moment-sensitive, has been accounted for. The strain sensed by SG1, SG2 and SG3 due to alignment errors was less than $5.4 \%$ of the designed sensitivity and was therefore neglected. To obtain true strain values the signals acquired by the data acquisition system via the strain gauge bridges were converted to strain at the particular nodes as follows. The strain gauge bridge equation is

$\frac{v_{o_{k}}}{v_{i_{k}}}=\frac{k_{g}}{4}\left(\varepsilon_{1}-\varepsilon_{2}+\varepsilon_{3}-\varepsilon_{4}\right)$

where $v_{o_{k}}$ and $v_{i_{k}}$ are the bridge output and supply voltages respectively and $k_{g}$ is the gauge factor of the gauge type used for a particular bridge. The full bridge was used to measure thrust and was congured such that $\varepsilon_{2}=\varepsilon_{4}=v \varepsilon_{z}$ and $\varepsilon_{1}=\varepsilon_{3}=-\varepsilon_{z}$, where $v=0.3$ was used as the Poisson $\square \mathrm{s}$ ratio with the convention that strains due to compression and elongation are negative and positive respectively. The half bridge was used to measure bending around the $x$ - and $y$-axis respectively and was congured such that $\varepsilon_{3}=\varepsilon_{4}=$ $0, \varepsilon_{1}=\varepsilon_{z}$ and $\varepsilon_{2}=-\varepsilon_{z}$. Since the signal measured by the data acquisition system was

$\varepsilon_{b i}=\varepsilon_{1}-\varepsilon_{2}+\varepsilon_{3}-\varepsilon_{4}$ for $i=1,2,3$

for the two half bridges measuring bending around the $y$-and $x$ - axis and the full bridge respectively in $[\mu \varepsilon]$. The strains at the three nodes selected for computation of the dynamic force at the tool tip therefore were 


$$
\begin{aligned}
& \varepsilon_{S G 3}=\left(\frac{1}{2(1+v)} \varepsilon_{b 3}\right) v \\
& \varepsilon_{S G 1}=0.5 \varepsilon_{b 1}-\varepsilon_{S G 3}\left(\frac{1}{v}\right) \text { and } \\
& \varepsilon_{S G 2}=0.5 \varepsilon_{b 2}-\varepsilon_{S G 3}\left(\frac{1}{v}\right) .
\end{aligned}
$$

\section{Wavelet packet based features}

\section{Wavelet transform}

A wavelet transform is an integral transform with the integrands being the signal and the mother wavelet function, or shifted and scaled versions of the latter. Changes in scale and time shift respectively result in dilation and translation of the wavelet function as described in Wu et al. (1996). They therefore have the prescribed property of localization in both time and frequency together with the smoothness property giving them the ability to convey essential information of the signal.

Unlike Fourier transform wavelet transforms have an analysing function of nite extent, which is one reason for its time localization property and makes it suitable to capture features of non-stationary signals such as cutting forces in turning processes. In this study however the wavelet packet transform where each packet is a particular linear combination of their parent wavelet is used.

\section{Wavelet packet analysis}

In wavelet analysis the signal is passed through two complementary lters, $G$ and $H$, splitting it into two signals, one with low frequency components through low-pass lter $H$, yielding an approximation coefcient vector and the other with high frequency components through high-pass lter $G$, yielding a detail coefcient vector. In wavelet packet analysis approximation and detail are split in a recurring way, again each into approximation and detail.

Each wavelet packet is part of a family of packets and forms an orthonormal basis of the parent wavelet as shown by Wickerhauser (1994). Using the notation of Wickerhauser (1994), the packet $\left\{W_{j n}(k): k \in Z\right\}$ is the sequence of the inner products of a function $x=x(t)$ square integrable, which in case of this study is the signal to be processed, with the basis functions $\gamma_{j n k}$ and is dened as

$$
W_{j n}(k) \stackrel{\text { def }}{=}\left\langle x, \gamma_{j n k}\right\rangle=\int_{R} \bar{x}(t) 2^{-j / 2} \gamma_{n}\left(2^{-j} t-k\right) d t
$$

where $\bar{x}(t)$ is the complex conjugate of $x(t)$.
The coefcient sequences $\left\{W_{j n}\right\}$ are then decomposed to the next level $j+1$ and satisfy the recursion relations

$W_{j+1,2 n}(k)=H W_{j n}(k)$

and

$W_{j+1,2 n+1}(k)=G W_{j n}(k)$.

If the wavelet packet decomposition is organized in a tree with the set of functions $W_{j n}=\left\{W_{j n k}(x): k \in Z\right\}$ representing the $\{j, n\}$ wavelet packet, the tree will be as shown in Fig. 6, where $W_{0,0}$ and $W_{1,1}$ are dened by the scaling function $\phi$ and the wavelet function $\psi$ respectively as

$W_{0,0}=\{\phi(x-k), k \in Z\}$

and

$W_{1,1}=\left\{\psi\left(\frac{x}{2}-k\right), \quad k \in Z\right\}$.

because one-dimensional wavelet analysis, such as in this application, is based on one scaling function $\phi$ and one wavelet function $\psi$, also called the parent wavelets. The Daubechies $1 \square 15$, Symmlets $2 \square 8$ and Coiflet $1 \square 5$ families of parent wavelets as used in this study, each have their particular scaling function and wavelet function.

Interpreted this means that the function space including $W_{0,0}$ spans the subspaces which include $W_{1,0}$ and $W_{1,1}$ where each subspace including $W_{j, n}$ is further split or decomposed into subspaces which include $W_{j+1,2 n}$ and $W_{j+1,2 n+1}$. The decomposition is organized in a tree and the scale parameter $j$ and frequency parameter $n$ are respectively labelling depth and position in the tree. The wavelet packet analysis in this study was aided by the Wavelet Toolbox from Mathworks (2006).

An indication of the relationship of $\phi$ and $\psi$ between different levels in the decomposition tree is based on the two-scale equation. For wavelet functions in this study a two-band system is considered and their scaling and translation is dened by

$\phi_{j, n, k}(t)=2^{-j / 2} \phi_{n}\left(2^{-j} t-k\right)$,

where $n \in N$ and $(j, k) \in Z^{2}$.

The relationship based on the two-scale equation with scale changes by any power of 2 , is given by Vetterli et al. (1995) as

$\phi(t)=2^{-j / 2} \sum_{k=0}^{L(j)-1} h^{(j)}[k] \phi\left(2^{-j} t-k\right)$

and also

$\psi(t)=2^{-j / 2} \sum_{k=0}^{L(j)-1} g^{(j)}[k] \phi\left(2^{-j} t-k\right)$ 


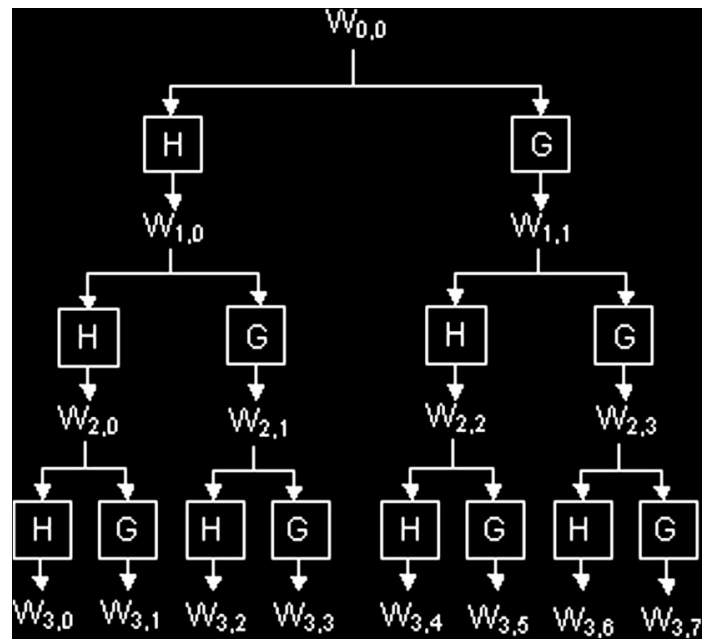

Fig. 7 Wavelet packet tree

where $h^{(j)}[k]$ and $g^{(j)}[k]$ are the coefcients of the iterated lters $H^{(j)}(z)$ and $G^{(j)}(z)$ respectively. For more detail on these iterated lters the reader is referred to the sections on $\square$ iterated Iter banks $\square$ and $\square$ multi-channel lter banks and wavelet packets $\square$ inVetterli et al. (1995).

Different criteria are available to determine the depth of decomposition in the tree. The entropy criterion was used here because it is commonly used in signal processing applications (Mathworks 2006). The maximum depth for which the entropy of more than $50 \%$ of the children coefcient sequences of a certain level is less than their corresponding parent sequence was chosen as the level or depth of decomposition.

The Shannon entropy relationship

$E(S)=-\sum_{k=1}^{N} S_{k}^{2} \log \left(S_{k}^{2}\right)$

was used here to determine the entropy of a signal $S$.

The coefcient sequences of each level of Fig. 7 each represent a frequency sub-band. If the original signal was sampled at $F_{S}=20 \mathrm{kHz}$ with a usable frequency $\frac{F_{S}}{2}=10 \mathrm{kHz}$ then in level $j=3$ there are $2^{j}$ sub-bands of equal width.

\section{Feature selection}

The decomposition level depends on the type of parent wavelet and the signal, i.e. tangential, feed or thrust force signal or the SSA strain signal. The signal was decomposed into a number of coefcient sequences representing above mentioned sub-bands. Coefcients for the detail of the decomposed signal are smaller than those of the approximation part, which represents the lower frequency sub-bands. Energy values of frequency sub-bands were considered for the removal of noise. The root-mean-square (RMS) values of each sub-band $\square$ s coefcient vector were taken as a measure of that particular sub-band $\square$ s energy. Frequency bands, which together had an energy sum below a threshold of $2 \%$ were discarded. This method of noise removal can be regarded as a type of threshold technique.

For TCM based on a strain-gauge instrumented tool holder the features are classied according to three degrees of freedom, namely the signal of a particular force component, the wavelet type and sub-band energy number. Regarding the TCM based on a SSA, features are classied according to only two degrees of freedom, namely the wavelet type and sub-band energy number. For the purpose of TCM the most wear sensitive features were selected as in Scheffer and Heyns (2004) by correlation using

$$
\rho=\frac{\sum_{i}\left(V_{B i}-\bar{V}_{B}\right)\left(\theta_{n i}-\bar{\theta}_{n}\right)}{\sqrt{\sum_{i}\left(V_{B i}-\bar{V}_{B}\right)^{2} \sum_{i}\left(\theta_{n i}-\bar{\theta}_{n}\right)^{2}}} .
$$

with $V_{B i}$ and $\theta_{n i}$ being ank wear and the corresponding $n$th feature at the $i$ th instant of measurement. Since the feature space is here dened by a component of the wavelet type and also the frequency sub-band component, the selection of a certain correlating feature would then determine by which wavelet and sub-band it is described.

For strain gauge based TCM the following two features, each described by three degrees of freedom, have been selected.

\begin{tabular}{lll}
\hline 1) & Force component & :tangential \\
Wavelet type & $:$ Symmlets 1 \\
Feature/Sub-band/ & $: \theta_{16} / 2344$ to $2500 \mathrm{~Hz} / 6$ \\
$\begin{array}{l}\text { Decomposition level } \\
\text { Force component }\end{array}$ & :feed \\
Wavelet type & $:$ Symmlets 8 \\
Feature/Sub-band/ & $: \theta_{1} / 0$ to $156 \mathrm{~Hz} / 6$ \\
& \\
\hline
\end{tabular}

For TCM based on a SSA the two features, each described by two degrees of freedom were selected as follows.

\begin{tabular}{lll}
\hline 1$)$ & Wavelet type & :Symmlets 4 \\
& Feature/Sub-band/ & $\theta_{1} / 0$ to $312 \mathrm{~Hz} / 5$ \\
& $\begin{array}{l}\text { Decomposition level } \\
\text { 2) }\end{array}$ & \\
Wavelet type & $:$ Daubechies 1 \\
& Feature/Sub-band/ & $: \theta_{40} / 6094$ to $6250 \mathrm{~Hz} / 6$ \\
& Decomposition level & \\
\hline
\end{tabular}

\section{Data processing}

\section{Design of experiments}

For the cutting parameters CS, DOC and FR, a full factorial design of experiment (DOE) was chosen, i.e. a number of $q=3$ levels for each cutting parameter so that the total number of experiments for the design was $3^{q}=27$. This 
Fig. 8 Monitoring with a time-delay neural network structure

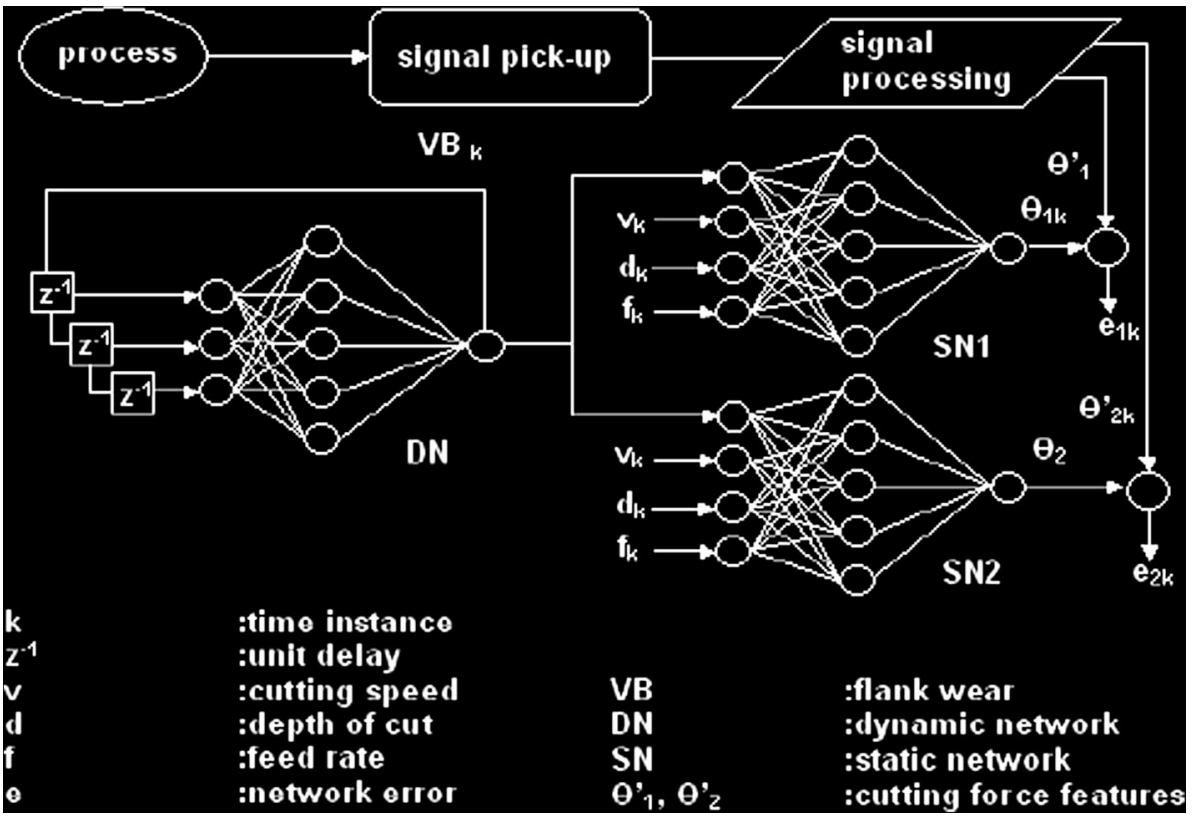

means that for every set of three experiments, one parameter was adjusted to three different levels, while the other two were kept constant. The experiment was also conducted according to DOE in order to obtain data, which allow for training the $\mathrm{NN}$ with the inuence of all the above-mentioned cutting parameters.

\section{Neural networks}

A NN-approach was used for the comparison of two ways of monitoring tool wear, because it has already successfully produced results that appeared in the literature and was mentioned in $\square$ Introduction $\square$ section. In the rst, features are obtained from strain gauge based sensing of the orthogonal cutting force components. In the second, tool wear monitoring is based on features available from a single strain signal that would be experienced by the SSA due to the cutting force at the tool tip.

A network structure as depicted in Fig. 8 was selected. This is based on a structure published by Sick (2002) and later also applied by Scheffer et al. (2003), and consists of a dynamic network (DN) with time-delayed inputs and a number of static networks (SN) corresponding to the number of features involved. All networks are of the feed-forward type with the rst layer consisting of tan-sigmoid neurons to provide non-linear properties and the output layer of a linear transfer function to allow for a wide range of output values. The architecture choice of the DN and the SN are 5-1 and $5-1$ respectively, resulting in 26 and 31 parameters for the DN and SN respectively.

The DN was trained online, using the Particle Swarm Optimisation Algorithm (PSOA) as in the work of Scheffer et al.
(2003). In this algorithm a complete set of $n$ network parameters (weights and biases) constitutes a multi-dimensional particle position, w, at a given instant in time. A swarm size of 25 is considered. This is a value used in Scheffer et al. (2003) and it was also found that a larger swarm population, such as 40 , did not improve the monitoring capabilities. The optimisation starts with the initialised space

$D=\left\{\boldsymbol{w} \in \Re^{n}: 1<w_{i}<1, \quad i=1 \ldots n\right\}$

and considers the optimisation problem

$\min g_{k}(\boldsymbol{w})=\sum_{j=1}^{m} e_{j k}$

where

$e_{j k}=\sqrt{\left(\theta \prime_{j k}-\theta_{j k}\right)^{2}}, \quad j=1 \ldots m$

with $\theta^{\prime}{ }_{j k}$ and $\theta_{j k}$ the $j$ th true and estimated signal features for a certain interval respectively at the $k$ th measuring instant.

The optimisation before each true time increment was performed for 40 pseudo time steps, $\tau$. The algorithm is briey described by the updating equations for velocity, $\hat{\boldsymbol{v}}$, of particle $i$ at pseudo time step $\tau+1$, namely:

$\hat{\boldsymbol{v}}_{\tau+1}^{i}=q \hat{\boldsymbol{v}}_{\tau}^{i}+c_{1} r_{1}\left(\boldsymbol{p}_{\tau}^{i}-\boldsymbol{w}_{\tau}^{i}\right)+c_{2} r_{2}\left(\boldsymbol{p}_{\tau}^{g}-\boldsymbol{w}_{\tau}^{i}\right)$

with $\boldsymbol{p}_{\tau}^{i}$ the best ever position of particle $i$ at time $\tau$ and $\boldsymbol{p}_{\tau}^{g}$ the global best position in the swarm at time $\tau$. In Eq. (33), $r_{1}$ and $r_{2}$ are random numbers chosen as

$H=\left\{r_{i} \in \Re: \quad 1<r_{i}<1, \quad i=1,2\right\}$,

and $c_{1}=c_{2}=2$ in order to keep the mean gain of each term equal to 1 . The inertia parameter, $q$, in Eq. (33) was initially 
set to 0.99 and then decreased linearly during optimisation. The updating equation for position, $\boldsymbol{w}$, is

$\boldsymbol{w}_{\tau+1}^{i}=\boldsymbol{w}_{\tau}^{i}+\hat{\boldsymbol{v}}_{\tau+1}^{i}$.

Due to the design of the experiment as well as the factors inuencing the process as mentioned in $\square$ Experimental procedure $\square$ section, limited data was available to train the SNs. Consequently, SN-training was done by means of Bayesian Regularisation, using the Neural Network Toolbox of Mathworks (2002b), to improve generalisation and prevent over-tting. In order to deal with the lack of training data an attempt was made to reduce the input space by applying principal component analysis (PCA) as described in Jackson (1991). However from the input space consisting of 27 entries of the inputs CS, DOC and FR, none of the inputs could be discarded because each of them contributed at least $30 \%$ to the total variance of the inputs. Discarding one of the cutting parameters could not perform a reduction of above mentioned input space. If the features are also seen as system inputs one cannot reduce the input space by discarding certain features because doing that one also has to discard SNs and this in turn will change the whole system.

The neural network structure including the number of parameters to be trained is the same for both TCM systems, i.e. the one based on processing of orthogonal force signals and the other which is based on processing of unidirectional strain signal. Therefore the estimating speed of tool wear is the same for both. It is approximately $60 \mathrm{~s}$ per data point while the cutting time intervals between data points is above $180 \mathrm{~s}$ for the cutting conditions of this work. The data processing was done on a Pentium ${ }^{\circledR} 4$ with $2.4 \mathrm{GHz}$ CPU.

\section{Results}

Selection of training sets

From the 27 tool insert data sets, $Q_{i}$, with $i=1, \ldots 27$ rst a selection $E_{2}$ based on two criteria was used to determine the relative performance of the two TCM systems under consideration. These two criteria are described as follows.

1) The correlation coefcient of the features with tool wear on an insert should be larger than 0.85 . Let the set which satises this criterion be called $E_{1}$, i.e.

$$
E_{1}=\left\{Q_{i}: \rho_{i}>0.85, \quad i=1, \ldots 27\right\}
$$

2) At least 4 of the data sets $Q_{i}$ with $i=1, \ldots 27$, which are elements of $E_{1}$ should be part of the above mentioned selection with an approximately equal representation of each of the three speed settings:

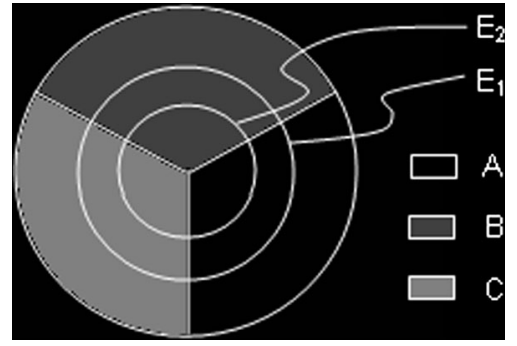

Fig. 9 Venn diagram of selected training set

$$
\begin{gathered}
E_{2}=\left\{Q_{i}: P\left(A / E_{1}\right) \approx P\left(B / E_{1}\right) \approx P\left(C / E_{1}\right)\right. \\
\left.\geq \frac{4}{27}, \quad i=1, \ldots 27\right\}
\end{gathered}
$$

where $P(J / K)$ means the probability of $J$ given $K$ and

$$
\begin{aligned}
& A=\left\{Q_{i}: C S=128.8 \mathrm{~m} / \mathrm{min}\right\}, \\
& B=\left\{Q_{i}: C S=119.8 \mathrm{~m} / \mathrm{min}\right\}, \\
& C=\left\{Q_{i}: C S=114.6 \mathrm{~m} / \mathrm{min}\right\} .
\end{aligned}
$$

This criterion was included since for the ranges of cutting parameters used in the experiment of this study, tool wear is more sensitive to changes in CS than to changes of any of the two other cutting parameters. This could be seen on the three-dimensional response surfaces of ank wear versus $\mathrm{CS}$ and $\mathrm{FR}$ and ank wear versus $\mathrm{CS}$ and DOC.

The selection of the training set can be illustrated in a Venn diagram as shown in Fig. 9 with $E_{2}$ as a subset of $E_{1}$.

In order to increase the statistical condence in the results of the relative performance of the two TCM systems a number of 9 out of 12 data sets, which were most sensitive to tool wear, were used for training and the remaining 3 sets were used for testing of the TCM systems to be compared. Each experiment (i.e. each data set) provided 6 training input patterns corresponding to the 6 instances of ank wear measurement.

\section{Hypothesis test}

In this hypothesis test the difference in mean errors $\delta \mu_{e r r}$ in the estimation of ank wear values between the two TCM systems are under consideration. The general requirement with respect to the TCM based on a linear combination of the orthogonal force components is that

$\delta \mu_{0}-l \leq \delta \mu_{\text {err }} \leq \delta \mu_{0}+l$, with $l=0.05$ and $\delta \mu_{0}=0$ 

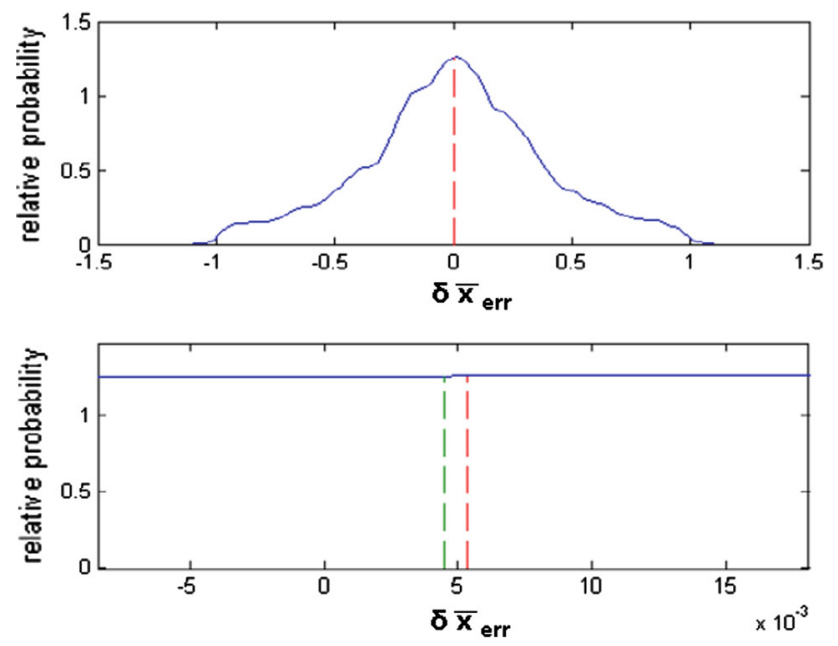

Fig. 10 Subplot 1: complete density of relative probability. Subplot 2: density of relative probability zoomed in on $5 \%$ condence interval

The difference in mean errors is dened as

$\delta \mu_{e r r}=\mu_{e r r S S A}-\mu_{e r r S G}$

where

$$
\begin{gathered}
\mu_{\text {err SSA }}=\frac{1}{N} \sum_{i=1}^{N}\left|V_{\text {Brue }_{i}}-V_{B S S A i}\right| \\
\mu_{\text {err } S G}=\frac{1}{N} \sum_{i=1}^{N}\left|V_{\text {true }_{i}}-V_{B S G i}\right|,
\end{gathered}
$$

with $N$ the number of measurements. Subscripts $S S A$ and $S G$ refer to the SSA $\square$ and the strain gauge-based TCM systems respectively for the remainder of this section.

The graph in Fig. 9 shows the relative probability of $\left\{\delta \bar{x}_{e r r}:-1<\delta \bar{x}_{e r r}<1\right\}$ where $\delta \bar{x}_{e r r}=\bar{x}_{e r r S S A}-\bar{x}_{e r r} S G$. All $\left|\delta \bar{x}_{e r r}\right| \geq 1$ were regarded as outliers. It can be seen that the difference in sample means has a clear central tendency of $\delta \bar{x}_{e r r} \approx 0.0218$ but a high variance, which is expected to be the result of process uncertainties. Here $\bar{x}$ refers to the sample mean. Since the value of $\delta \bar{x}_{e r r} \approx 0.0049$ already falls in the required interval specied in Eq. ( 38 ) it is further determined by hypothesis testing with what probability will it be found at the value $\delta \mu_{1}=0.0048$, i.e. close to $\delta \bar{x}_{e r r} \approx 0.0049$ (Fig. 10).

The hypothesis to be tested here is as follows. For the null-hypothesis to be true there should be no difference in the mean errors of ank wear estimation between the TCM systems, i.e.

$$
\begin{aligned}
H_{0}: \delta \mu_{\text {err }} & =\delta \mu_{1} \\
H_{1}: \delta \mu_{\text {err }} & \neq \delta \mu_{1}
\end{aligned}
$$

with significance level of $\alpha=0.95$, in order to keep the probability of a type II error $\beta$, for which $\beta \rightarrow 1-\alpha$ if $\delta \mu_{e r r} \rightarrow \delta \mu_{1}$ as low as possible. A type II error occurs if
$H_{0}$ is false and is not rejected but if $H_{1}$ which would then be true is wrongly rejected instead.

Generally it is not known on which side of zero $\delta \mu_{\text {err }}$ lies, a two-tailed test for the variable of differences between means $\delta \mu_{\text {err }}$, is therefore done. The test of the statement in Eq. $(42 a, b)$ is based on the statistic

$t_{0}=\frac{\delta \bar{x}_{e r r}-\delta \mu_{1}}{S / \sqrt{n}}$

where $n$ is the sample size and $S$ the standard deviation of the sample.

For a sample of $n=3,310$ and $S=0.383$, which result in $t_{0}=0.015$. With a significance level $\alpha=0.95$ and using a $\mathrm{t}$-distribution, the corresponding interval $\left[-t_{\alpha / 2 ; n-1}, t_{\alpha / 2 ; n-1}\right]$ is determined using the t-distribution calculator at http:// stattrek.com/Tables/t.aspx as $[-0.063,0.063]$. Since $t_{0}$ is bracketed by this interval $H_{0}$ is accepted.

The significance of having made an error with this acceptance is $\beta \approx 0.05$. This is the probability of a type II error, which would be the failure to reject $H_{0}$ if it is false. Therefore $H_{0}$ as formulated in Eq. (42a, b) is accepted. This also satises the general requirement set in Eq. ( 38 ).

\section{Accuracy of monitoring systems}

This section is organized in two parts. The rst one contains a description of the procedure by which the accuracy of above mentioned two systems was measured. The contents of the second part describe the room found to improve the accuracy of the two systems after they were trained using different standard deviations of error for tool wear data.

\section{Current accuracy}

The accuracy of these monitoring systems was tested by using any 24 from the total of 27 data sets for training of the networks. For the testing of the networks the remaining 6 data sets were used. The only condition to this selection was that each of the three cutting parameter settings would be represented an equal number of times in the data sets used for testing. Each data set contributed 6 measurement points and in neural network terminology therefore 6 patterns.

The average accuracy of the remaining tool wear estimation expressed as the percentage of the error with respect to the measured values for tool wear for orthogonal TCM and unidirectional TCM is given in Table 2. The cutting parameter values given in $\square$ Experimental procedure $\square$ section correspond to the notation in Table 2.

Human decision making may me incorporated in condition monitoring algorithms such as dealt with in Gajate et al. (2012). For the NN-algorithm as used in this work, decision-making can be avoided with the availability of a 
Table 2 Flank wear error statistics a Estimated data points of set $D$ were discarded (see text)

\begin{tabular}{|c|c|c|c|c|}
\hline \multirow{2}{*}{$\begin{array}{l}\text { Data set/s for } \\
\text { testing with } 24 \\
\text { training data sets } \\
\end{array}$} & \multicolumn{2}{|c|}{$\begin{array}{l}\text { All combinations, that have all } \\
\text { cutting parameter values } \\
\text { represented in a test set } \\
\text { consisting of } 3 \text { data sets }\end{array}$} & \multicolumn{2}{|c|}{$\begin{array}{l}\left(\mathrm{CS}_{1}, \mathrm{DOC}_{2}, \mathrm{FR}_{2}\right),\left(\mathrm{CS}_{2}, \mathrm{DOC}_{1}, \mathrm{FR}_{1}\right), \\
\left(\mathrm{CS}_{3}, \mathrm{DOC}_{3}, \mathrm{FR}_{3}\right)\end{array}$} \\
\hline & Orthogonal & Unidirectional & Orthogonal & Unidirectional \\
\hline SD & 0.23 & 0.248 & 0.194 & 0.258 \\
\hline $\begin{array}{l}\text { Mean error } \mu_{e r r}: \\
\quad \text { current accuracy }\end{array}$ & $32.0 \%$ & $33.3 \%$ & $31.3 \%$ & $36.7 \%$ \\
\hline $\begin{array}{l}\text { Mean error } \mu_{\text {err }}: \\
\text { improved accuracy }\end{array}$ & $\square$ & $\square$ & $18.2 \%$ & $16.4 \%$ \\
\hline
\end{tabular}

much larger number of training data. In Table 2 reasoning however is used to discard estimation errors greater than $100 \%$, since these could be an indication of insert breakage rather than expected wear. In the light of measured ank wear increments between consecutive measurement instants of between 1 and $69 \%$ this is a justiable limit of exclusion. The category of insert breakage is beyond the scope of this work and is regarded as an error by the monitoring system rather than an estimation of expected ank wear. Therefore the estimated data points in set $D=\left\{\mu_{e r r}:-1>\mu_{\text {err }}>1\right\}$ have been discarded for the gures given in Table 2 . However the absolute maximum and minimum values of estimated ank wear VB are shown in Figs. 11 and 12 except for a $0.6 \%$ of the data points which were regarded as outliers. Figures 11 and 12 show the results for orthogonal and unidirectional TCM respectively for a test data set as specied in the right half in Table 2.

Figure 13 shows the range of measurement errors of true ank wear with the range as well as true ank wear averaged over all 27 data sets. This error ranges between 3.7 and $17.7 \%$ of the corresponding true measured ank wear values and is due to the limited accuracy of the microscope set-up for ank wear measurement (see Table 1)

The results in Table 2 and Figures 11 and 12 must be interpreted against this background and that only a small number of input patterns were available for training of the SNs.

To get an impression about the improvement of accuracy of a multi-layer perceptron type of $\mathrm{NN}$ the interested reader is referred to Wang et al. (2008). The next section shows what room there is to improve the accuracy of the $\mathrm{NN}$ used in this work without changing its structure.

\section{Improved accuracy}

Figure 14 shows the sensitivity of the monitoring systems as a standard deviation of the error of the estimated tool wear versus the standard deviation of the error of tool wear values which were used for training purposes. The cutting parameter settings of the training and test sets that were used to nd the improved accuracy were the same as used to nd the current accuracy of the two systems described in the section under
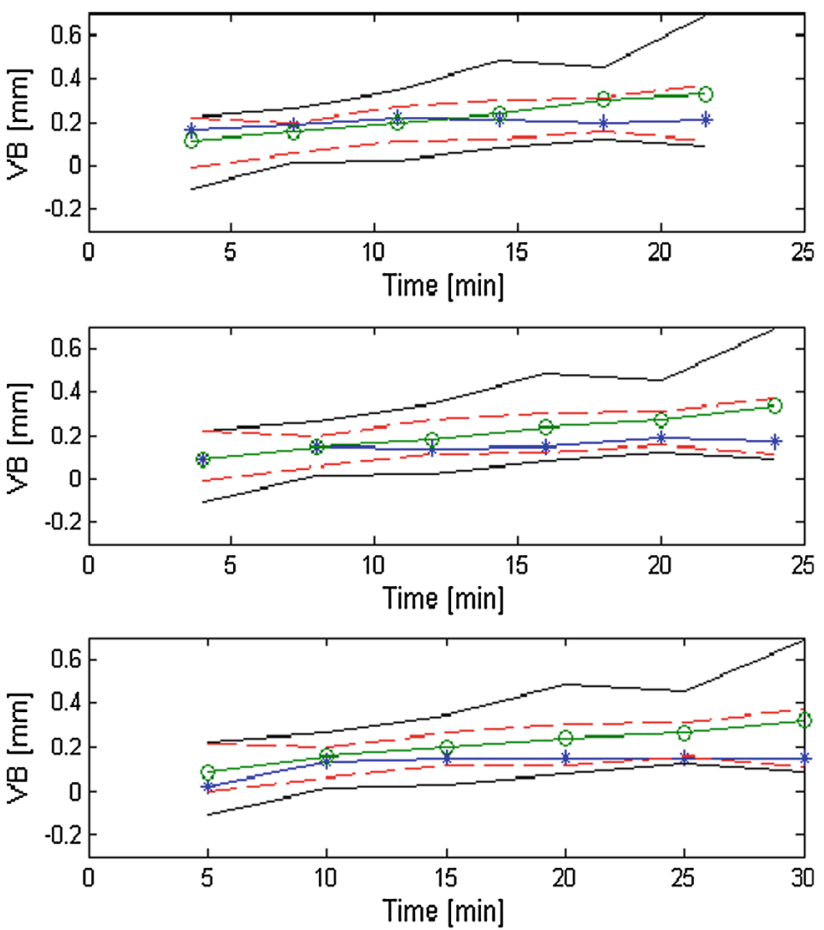

Fig. 11 Estimated VB (asterisk) compared with measured VB (circle) using orthogonal TCM and SD (dashed red) as well as range limits (solid black) of estimated VB (Color gure online)

the heading current accuracy. However the reference values for tool wear used here were determined by means of models, which in turn were obtained through full quadratic regression at 6 linearly spaced time instances of the cutting process from $t=300$ to $1,296 \mathrm{~s}$. The tool wear data for this regression $\mathrm{t}$ was obtained by interpolating the tool wear values for each cutting parameter setting at above mentioned time instances. The resulting 6 models then yielded the tool wear reference values as functions of the three cutting parameters, CS, DOC and FR.

Figure 14 shows that a reduction of standard deviation on the error of the input training data from $S_{V B}$ err tr $=0.015$ down to zero would result in a standard deviation of as low as $S_{V B \text { err est }}=0.114$ in the error of tool wear estimation 

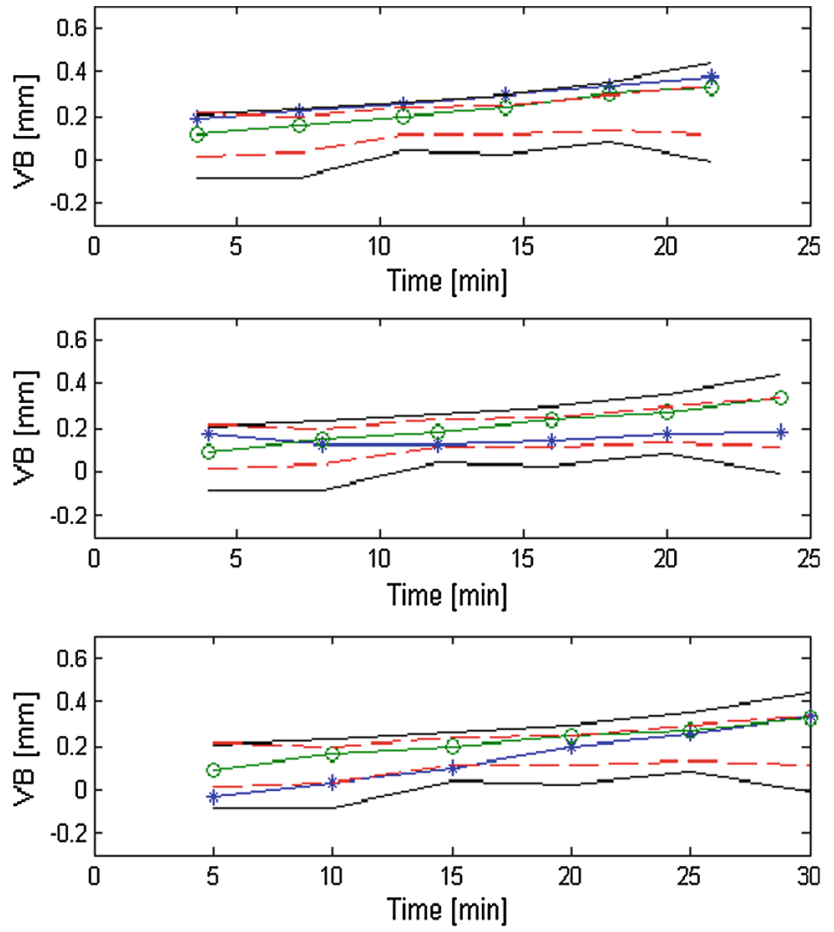

Fig. 12 Estimated VB (asterisk) compared with measured VB (circle) using unidirectional TCM and SD (dashed red) as well as range limits (solid black) of estimated VB (Color gure online)

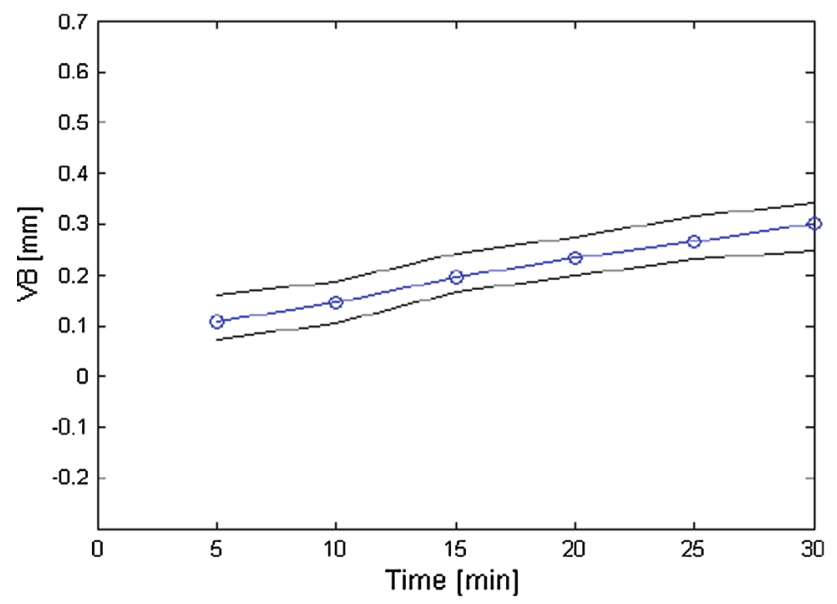

Fig. 13 Range of measurement errors of true ank wear

at the output side of system 1 and system 2 instead of the $S_{V B \text { err est }}=0.22$ and 0.33 before such a reduction. A $S_{V B \text { err est }}=0.114$ due to above mentioned reduction of error standard deviations in input training tool wear data would then correspond to an average tool wear estimation error of 18.2 and $16.4 \%$ for orthogonal TCM and unidirectional TCM respectively. These percentages may be improved even further by using data, which corresponds to at least twice as many patterns for training of the networks and improved noise reduction in the signals sensed from the cutting process.

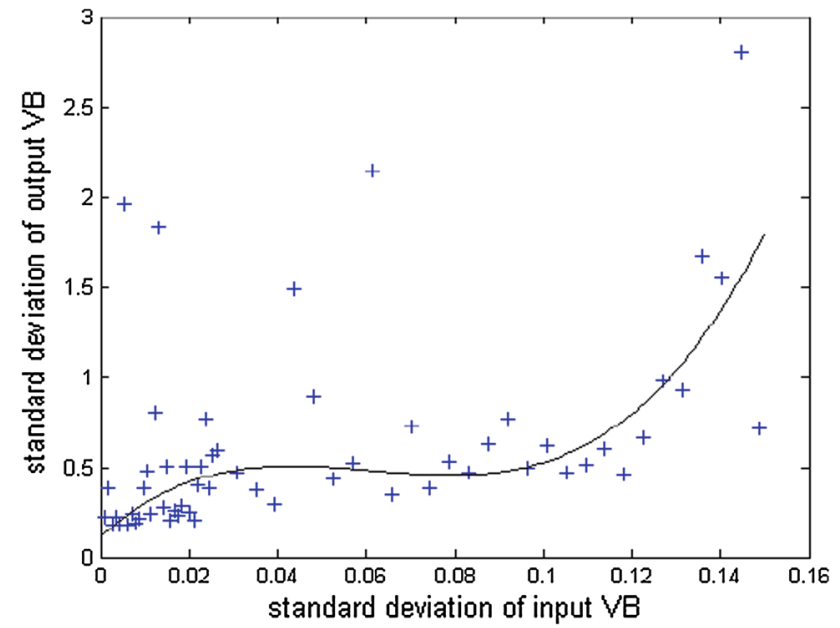

Fig. 14 Standard deviation of estimated (output) VB values versus $\mathrm{SD}$ of VB values used for training purposes (input) determined with 3rd order robust regression $\mathrm{t}$

\section{Conclusion}

Cutting-force measurements were used to investigate whether it would be feasible to utilise a signal, which is a linear combination of the orthogonal force components at the tool tip. The feedback signal, which a semi-embedded SSA provides in an active vibration control system, was mentioned as specic case where this signal would be used for condition-monitoring purposes. A derivation of the models linking strain to dynamic force on the specic tool holder, which was used for this study, was therefore given.

Under manual machining conditions with non-stationary cutting force signals a wavelet packet analysis was followed to generate tool wear sensitive features of the signals to be used in the two different monitoring systems under consideration. One system was based on the processing of orthogonal force components and the other on the processing of a linear combination of these components.

A loss of accuracy for TCM based on a unidirectional sensed signal as compared to the orthogonal reference case would have been plausible. However a hypothesis $t$ test showed that the probability of a difference of ank wear estimation error by less than 5 percentage points for these two monitoring systems is 0.95 when using a time-delayed neural network algorithm. Based on information about the sensitivity of the standard deviation of tool wear estimation error versus the standard deviation of the tool wear error for network training an accuracy the two monitoring systems can be indicated. It is indicated as 18.2 and $16.4 \%$ for the TCM system based on processing of orthogonal force components and the TCM system based on the processing of unidirectional strain respectively. This justies further research into TCM using signals, which are a linear combination of the 
orthogonal components sensed as in the specicapplication described in this study.

Acknowledgments The continued funding of this project by the National Research Foundation of South Africa is gratefully acknowledged. BHF appreciates the major support to nance his maintenance in Pretoria from his father Eckhart Freyer. BHF also wishes to thank his former colleague Professor Sukhomay Pal for a discussion on the use of wavelets.

\section{References}

Abouletta, O. B., \& M $\square$ dl, J. (2001). Surface roughness prediction based on cutting parameters and tool vibrations in turning operations. Journal of Materials Processing Technology, $118(1 \square$ 3), $269 \square 277$.

Anderson, E. H., \& Hagood, N. W. (1994). Simultaneous piezoelectric sensing/actuation: Analysis and application to controlled structures. Journal of Sound and Vibration, 174(5), $617 \square 639$.

Audy, J. (2006). An appraisal of techniques and equipment for cutting force measurement. Journal of Zhejiang University-Science A, $7((11), 1781 \square 1789$.

Bahre, D., Rosenberger, U. \& Warnecke, G. (1992). Monitoring of cutting processes by vibration analysis: Exemplary applications in grinding and turning processes. Sensors and Signal Processing for Manufacturing; ASME, Production Engineering Division (Publication) (Vol. 55, pp. 65 $\square 80$ ).

Balm $\square$ s, E.\& Lecl $\square$ re, J.-M. (2003). Structure dynamics toolbox, for use with MATLAB ${ }^{\circledR}$, SDTools, User $\square$ s guide version 5.1.

Bonifacio, M. E. R., \& Diniz, A. E. (1994). Correlating tool wear, tool life, surface roughness and tool vibration in nish turning with coated carbide tools. Wear, 173(1 $\square 2)$, 137 $\square 144$.

Chen, S.-G., Ulsoy, A. G., \& Koren, Y. (1993). Computational stability analysis of chatter in turning. ASME Dynamic System Control Division Publications, 50, $107 \square 111$.

Cho, D.-W., Lee, S. J., \& Chu, C. N. (1999). The state of machining process monitoring research in Korea. International Journal of Machine Tools\& Manufacture, 39, 1697 $\square 1715$.

Choudhury, S. K., \& Bartarya, G. (2003). Role of temperature and surface nish in predicting tool wear using neural network and design of experiments. International Journal of Machine Tools\& Manufacture, 43, 747 $\square 753$.

Del Pino, S., \& Pironneau, O. (2008). FreeFEM3D documentation, version 1.0pre11. http://www.freefem.org, May 3, 2012.

Dorf, R. C., \& Bishop, R. H. (2005). Modern control systems (10th ed.). Upper Saddle River, New Jersey: Pearson Education, Inc..

Frey, P. J. (2001). MEDIT: An interactive visualization software. Institut National de Recherche en Informatique et en Automatique, Rapport technique no 0253, Th $\square$ me 4.

Freyer, B. H., Theron, N. J., \& Heyns, P. S. (2008). Simulation of tool vibration control in turning, using a self-sensing actuator. Journal of Vibration and Control, 14(7), 999 $\square 1019$.

Gajate, A., Haber, R., del Toro, R., \& Vega Bustillo, A. (2012). Tool wear monitoring using neuro-fuzzy techniques a comparative study in a turning process. Journal of Intelligent Manufacturing, 23, 869 $\square 882$.

Geuzaine, C., \& Remacle, J.-F. (2009), Gmsh: A three-dimensional nite element mesh generator with built-in pre- and post-processing facilities. International Journal for Numerical Methods in Engineering, 79(11), 1309 $\square 1331$ http://geuz.org/gmsh/,3.5.2012.

Jackson, J. E. (1991). A user $\square$ s guide to principal components New York: Wiley.

Jemielniak, K., Kwiatkowski, L., \& Wrzosek, P. (1998). Diagnosis of tool wear based on cutting forces and acoustic emission measures as inputs to a neural network. Journal of Intelligent Manufacturing, 9, 447 $\square 455$.

Li, C.-J., \& Ulsoy, A. G. (1999). Precision measurement of tool-tip displacement using strain gages in precision exible line boring. In American Society of Mechanical Engineers, Dynamic Systems

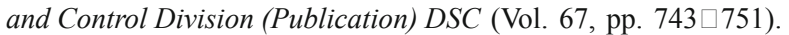

Liu, Q., \& Altintas, Y. (1998). On-line monitoring of ank wear in turning with multi-layered feed-forward neural network. International Journal of Machine Tools\& Manufacture, 39, $1945 \square 1959$.

Lundholm, T., Yngen, M., \& Lindstr $\square$ m, B. (1988). Advanced process monitoring $\square$ A major step towards adaptive control. Robotics and Computer Integrated Manufacturing, 4(3-4), 413ロ421.

Mathworks Inc. (2002a). User $\square$ s guide of control system toolbox, MAT$L A B$ R13. Natick: The MATHWORKS Inc.

Mathworks Inc. (2002b). User $\square s$ guide of neural network toolbox, MAT$L A B$ R13. Natick: The MATHWORKS Inc.

Mathworks Inc. (2006). User $\square s$ guide of wavelet toolbox, MATLAB R2006. Natick: The MATHWORKS Inc.

Mitsubishi. (2007). General catalogue 2007 $\square 2008$, Mitsubishi Materials, C003B.

Pal, S., Heyns, P. S., Freyer, B. H., Theron, N. J., \& Pal, S. K. (2011). Tool wear monitoring and selection of optimal cutting conditions with progressive tool wear effect and input uncertainties. Journal of Intelligent Manufacturing, 22(4), 491 $\square 504$.

Pisoni, A. C., Santolini, C., Hauf, D. E., \& Dubowsky, S. (1995). Displacements in a vibrating body by strain gauge measurements. In Proceedings of the 13th international modal analysis conference, Nashville, TN, February.

Purushothaman, S. (2010). Tool wear monitoring using articial neural network based on extended Kalman lter weight updation with transformed input patterns. Journal of Intelligent Manufacturing, 21, 717 $\square 730$.

Seah, K. H. W., Li, X., \& Lee, K. S. (1995). The effect of applying coolant on tool wear in metal machining. Journal of Materials Processing Technology, 48, 495 $\square 501$.

Scheffer, C., \& Heyns, P. S. (2004). An industrial tool wear-monitoring system for interrupted turning. Mechanical Systems and Signal Processing, 18, 1219 $\square 1242$.

Scheffer, C., Kratz, H., Heyns, P. S., \& Klocke, F. (2003). Development of a tool wear-monitoring system for hard turning. International Journal of Machine Tools\& Manufacture, 43, 973 $\square 985$.

Sharma, V. S., Sharma, S. K., \& Sharma, A. K. (2008). Cutting tool wear estimation for turning. Journal of Intelligent Manufacturing, 19, 99 $\square 108$.

Sick, B. (2002). On-line and indirect tool wear monitoring in turning with articial neural networks: A review of more than a decade of research. Mechanical Systems and Signal Processing, 16(4), 487ロ 546.

Skogestad, S., \& Postlethwaite, I. (2001). Multivariable feedback control: Analysis and design. Chichester, England: Wiley.

Swantek, S. D., Wicks, A. L., \& Wilson, L. T. (2001). SEM proceedings of the 19th international modal analysis conference (IMAC $X I X)$, Orlando, FL, USA, February $5 \square 9$.

Vetterli, M., \& Kovacevic, J. (1995). Wavelets and subband coding. Englewood Cliffs, New Jersey: Prentice-Hall, Inc..

Wang, X., Wang, W., Huang, Y., Nguyen, N., \& Krishnakumar, K. (2008). Design of neural network-based estimator for tool wear modeling in hard turning. Journal of Intelligent Manufacturing, 19, $383 \square 396$.

Wickerhauser, M. V. (1994). Adapted wavelet analysis from theory to software. Piscataway, New Jersey: IEEE Press.

Wu, Y., \& Du, R. (1996). Feature extraction and assessment using wavelet packets for monitoring of machining processes. Mechanical Systems and Signal Processing, 10(1), $29 \square 53$. 